\title{
Magic XPI Integration Component - EDI
}

Prof.(Dr.) S. T. Patil ${ }^{*}$, Surabhi Jadhav

Department of Computer Engineering, Vishwakarma Institute of Technology, Pune, Maharashtra, India

\section{ABSTRACT}

\section{Article Info}

Volume 7, Issue 3

Page Number: 611-614

Publication Issue :

May-June-2021

Article History

Accepted : 10 June 2021

Published : 15 June 2021
The electronic data exchange between B2B partners through EDI (Electronic Data Interchange) enables your company to continuously digitize and automate business processes. This results in more efficiency and easy, as well as cost savings as per document, but requires the support of different formats, message standards, and communication protocols. Organization offers a standard solution for B2B integration and electronic data exchange with the Magic EDI Service Platform. Whether you are a customer, supplier you can now exchange data electronically with all your partners.

Keywords : Communication Standards, B2B, EDI Service

\section{INTRODUCTION}

EDI is a computer-to-computer exchange of business documents in a standard electronic format between any discrete entities-customers, partners, branches, subsidiaries, or departments. By changing the document from a manual exchange of business documents to one that is fully automated with EDI, organizations see multiple benefits from reduced costs, faster document processing, fewer errors, and smoother relationships with partners and consumer.

EDI describes machine-to-machine exchange between application systems using electronic connection standards (such as AS2, FTP, X.400, etc.) Machine to machine exchange is the key here. EDI replaces any kind of manual processing of documents via fax, mail, or email with a fully automated workflow that does not include manual actions.

EDI enables employees to store business documents (such as invoices, orders, and delivery bills) internally in the system or application, automatically exchanging data electronically via a secure and encrypted network with partners, vendors, or other departments within the organization.

EDI workflows are fully automated and real-time. Documents can flow straight through to the appropriate application on the receiver's host or application (e.g., the Order Management System) and processing can begin immediately.

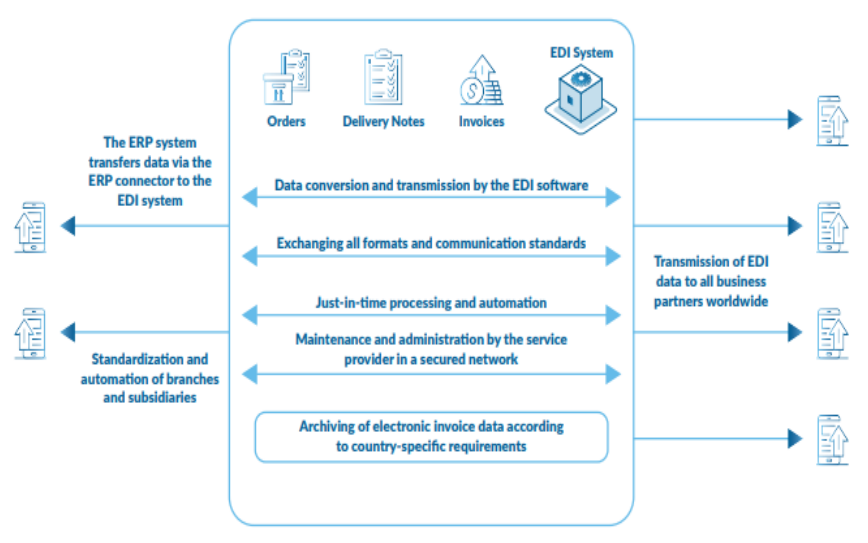




\section{METHODS AND MATERIAL}

\section{The basics of EDI communication}

It must be specified which message and communication standard is used to exchange the structured data on a secure path in both directions. In most cases, a business partner specifies the message standards and the communication path can be chosen accordingly. With communication standards, it is essential to convert the data into the standard preferred or supported by the partner. With EDI, once the communication standard is agreed upon, all that is usually required is a firewall white listing and short communication between the business partners to initialize the exchange.

\section{Common EDI Message Standards}

There are many EDI standards some of which address the needs of specific industries or regions. An overview of the common standards and their explanations follows below:

\begin{tabular}{|l|l|}
\hline XML & $\begin{array}{l}\text { The Extended Mark-up Language is a } \\
\text { cross-industry, international standard for } \\
\text { electronic data in business transactions, } \\
\text { in which the values can be given their } \\
\text { own names. }\end{array}$ \\
\hline CSV & $\begin{array}{l}\text { A comma-separated value is a cross- } \\
\text { sector, international standard that has } \\
\text { values separated by commas. }\end{array}$ \\
\hline
\end{tabular}

\section{Common Communication Standards}

Here are the most important standards for the transmission of electronic data that are used in EDI communication.

\begin{tabular}{|l|l|}
\hline HTTP & $\begin{array}{l}\text { The Hypertext Transfer Protocol } \\
\text { (Secure) transfers files via the } \\
\text { Internet/other networks. Often used for } \\
\text { payment transactions \& sensitive data }\end{array}$ \\
\hline FTP & $\begin{array}{l}\text { The File Transfer Protocol is a standard } \\
\text { network protocol for exchanging data }\end{array}$ \\
\hline
\end{tabular}

\begin{tabular}{|l|l|}
\hline & between client and server applications. \\
\hline SMTP & $\begin{array}{l}\text { Simple Mail Transfer Protocol. A } \\
\text { classical Email dispatch procedure, } \\
\text { which is also used for data exchange }\end{array}$ \\
\hline POP3 & $\begin{array}{l}\text { The abbreviation POP3 stands for Post } \\
\text { Office Protocol 3. is mostly used for } \\
\text { sending e-mails and also partly for } \\
\text { electronic data exchange. }\end{array}$ \\
\hline IMAP & $\begin{array}{l}\text { The abbreviation for Internet Message } \\
\text { Access Protocol, stands for a classic } \\
\text { procedure from the email dispatch, } \\
\text { which is also used for the transfer of } \\
\text { business documents and other data. }\end{array}$ \\
\hline
\end{tabular}

III. Plug \& Play principle

Once you connect your business processes to the Magic EDI platform, you can immediately exchange data with business partners and systems.

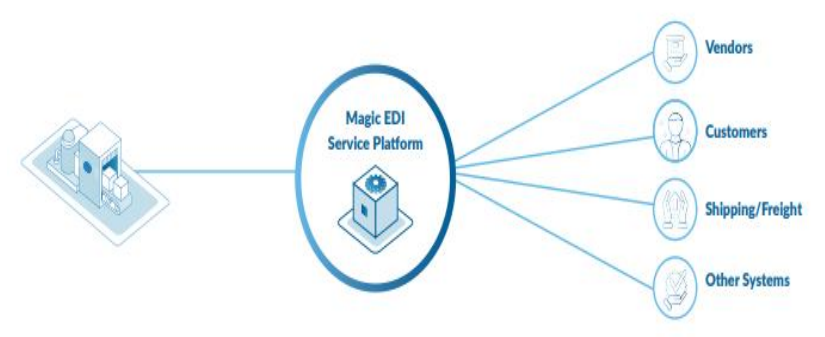

\section{Overview illustration for electronic data exchange}

Now you are familiar with most data formats and communication protocols. Our On Demand solution shows how to manage such a huge amount of requirements simply with a B2B solution and the variety of possibilities: 


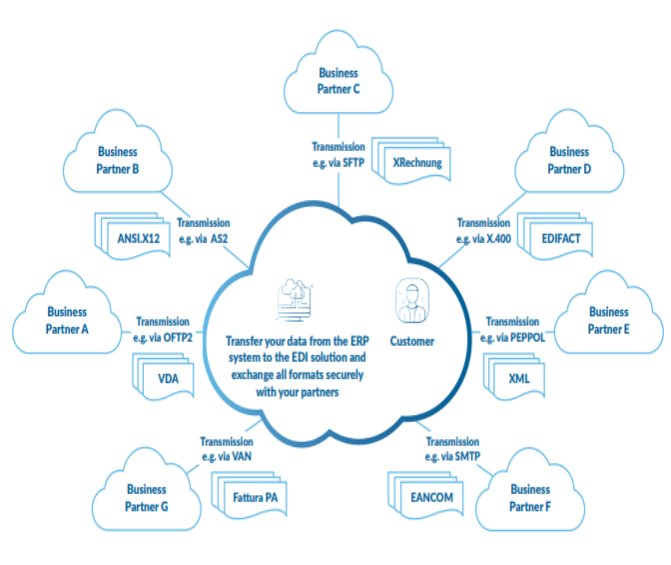

\section{EDI implementation}

For some enterprises, EDI can be difficult to implement. One reason is the need to keep pace with shifting government regulations, standards and updates. It is also inherently complex, as it needs to accommodate the complexities of global business needs. For example, each trading partner in a B2B network can present individual requirements. Even though two partners may agree on which EDI document to use, each can have unique formatting requirements that need to be supported. These factors, and others, have led many organizations to outsource their EDI solutions.

Whether in-house or outsourced, there are some basic conditions, capabilities and resources needed to implement EDI effectively. In addition to factors such as agreement on document types, secure transmission methods, and requisite hardware and software, an effective EDI implementation should consider:

\section{Translation or mapping software}

This type of transformation software takes fields such as names, addresses, currency amounts, part numbers and quantities, and maps them from business application formats into standardized documents and vice versa.

\section{Batch enveloping or de-enveloping capabilities}

These capabilities support large EDI message batches by enabling senders and receivers to wrap and unwrap transactions. The transactions can then be grouped from or split into several divisions or areas of a trading associate's business.

\section{Message routing mechanisms}

After a message is de-enveloped, routing mechanisms are required to sort the messages for different groups and deliver them to the appropriate targets. Message transformation may also be required to get the message into the correct format for its destination.

\section{Trading partner agreements (TPA)}

TPA clarifies terms and conditions, establishes standards for business documents and defines communications and business protocols between trading partners.

\section{FUTURE SCOPE}

EDIwill be the core document exchange capability to support innovations such as the Internet of Things (IoT), blockchain and artificial intelligence (AI). Future EDI will use:

IoT sensors incorporated into a shipment's packaging and tied to periodic EDI 214 messages to improve package condition visibility in near real time.

Block chain technology underpinning EDI information flows for shipments to offer a shared version of the truth that can quickly resolve and even avoid chargeback disputes.

An AI agent that monitors all relevant events and information connected to a shipment and can 
identify a non-compliant event. AI agents can also determine if a reshipment is required, analyse the most efficient source of replacement, initiate a new shipment and accept an authorized return.

\section{REFERENCES}

[1]. The Future of EDI: An IBM point of view

[2]. Electronic data interchanges, (link resides outside ibm.com)" Wikipedia

[3]. "EDI and B2B basics, (link resides outside ibm.com)" Sriniedibasics, 20 Aug 2011

[4]. "The future of EDI: An IBM point-of-view (PDF, 411 KB)" IBM Watson Supply Chain, Oct 2018

[5]. Seven key drivers for modernizing your B2B architecture $\mid \mathrm{I}$

[6]. What is B2B integration?|IBM

[7]. B2B collaboration solutions | IBM

\section{Cite this article as :}

Prof.(Dr.) S. T. Patil, Surabhi Jadhav, "Magic XPI Integration Component - EDI ", International Journal of Scientific Research in Computer Science, Engineering and Information Technology (IJSRCSEIT), ISSN : 2456-3307, Volume 7 Issue 3, pp. 611-614, May-June 2021. Available at doi : https://doi.org/10.32628/CSEIT2173133 Journal URL : https://ijsrcseit.com/CSEIT2173133 